\title{
Dual-Band Compact Planar Antenna for a Low-Cost WLAN USB Dongle
}

\author{
Maurício Henrique Costa Dias, ${ }^{1}$ Bruno Roberto Franciscatto, ${ }^{2}$ \\ Hans Adel, ${ }^{3}$ and Tan-Phu Vuong ${ }^{2}$ \\ ${ }^{1}$ Military Institute of Engineering, 22290-270 Rio de Janeiro, RJ, Brazil \\ ${ }^{2}$ Grenoble Institute of Technology, 38000 Grenoble, France \\ ${ }^{3}$ Fraunhofer Institute for Integrated Circuits, 90411 Nuremberg, Germany \\ Correspondence should be addressed to Maurício Henrique Costa Dias; mhcdias@ime.eb.br
}

Received 4 February 2014; Revised 18 April 2014; Accepted 21 April 2014; Published 28 May 2014

Academic Editor: Yingsong Li

Copyright (C) 2014 Maurício Henrique Costa Dias et al. This is an open access article distributed under the Creative Commons Attribution License, which permits unrestricted use, distribution, and reproduction in any medium, provided the original work is properly cited.

\begin{abstract}
Among the present technologies for WLAN devices, USB dongles still play a noticeable role. One major design challenge regards the antenna, which unavoidably has to comply with a very small volume available and sometimes should also allow multiband operation. In this scope, the present work discusses a dual-band WiFi compact planar IFA-based antenna design for a low-cost USB dongle application. Like most of the related published solutions, the methodology for deriving the present proposition was assisted by the use of an antenna analysis software. A prototype was assembled and tested in order to qualify the radiator design. Practical operation conditions were considered in the tests, such as the influence of the dongle case and the effect of the notebook itself. The results complied with the design constraints, presenting an impedance match quite stable regardless of the stick position alongside a laptop base.
\end{abstract}

\section{Introduction}

USB sticks or dongles represent a low-cost and simple way to provide network access to older desktops and notebooks or to computers with broken WiFi card. In the scope of wireless LAN device technology development, they still draw some attention, especially regarding the radiator design. The typical aesthetic constraints force the device to be small. Therefore, compact antenna design techniques are unavoidable.

Many different approaches have been reported so far, mainly along the past decade. As a general basis for this kind of device, practical radiator designs only consider the availability of a small percentage of the dongle volume, since most of it must be reserved for the electronic circuitry. The constraints vary among the different solutions proposed, sometimes allowing the use of the available space more effectively. 3D meandered shaped radiators or multilayered printed circuit board (PCB) fit well in such cases; see, for instance, $[1,2]$. On the other hand, low-cost designs call for the use of single-layered PCB, restraining the antenna to be at most " $2.5 \mathrm{D}$ " shaped, with vias or other short connections between the front and the back planes [3]. Sometimes, not even a back metal plane is present, leaving no choice for the antenna but to be planar.

WiFi dongles may be either single or dual-banded. The 2.4-2.48 and 5.15-5.85 GHz ISM bands cover such networks, with slight variations on the precise frequency limits from region to region [4]. The most recent issues of the WiFi standard include the possibility of MIMO system operation, and such feature is quite an active research field in the present scope [5-8]. There are also some attempts to derive multiband antennas for USB dongles to allow not only WiFi operation but also the access to other systems as well, such as LTE band $13(0.746-0.787 \mathrm{GHz})$ [8], GSM $(0.88-0.96 \mathrm{GHz})$ [9], UMTS $(1.92-2.17 \mathrm{GHz})$ [8-10], WiBro $(2.3-2.39 \mathrm{GHz})$ [813], LTE/WiMax (2.5-2.7 GHz) [7, 9-12], S-DMB (2.6052.655 GHz) [9-12, 14], or WiMax (3.4-3.6 GHz) [15]. 


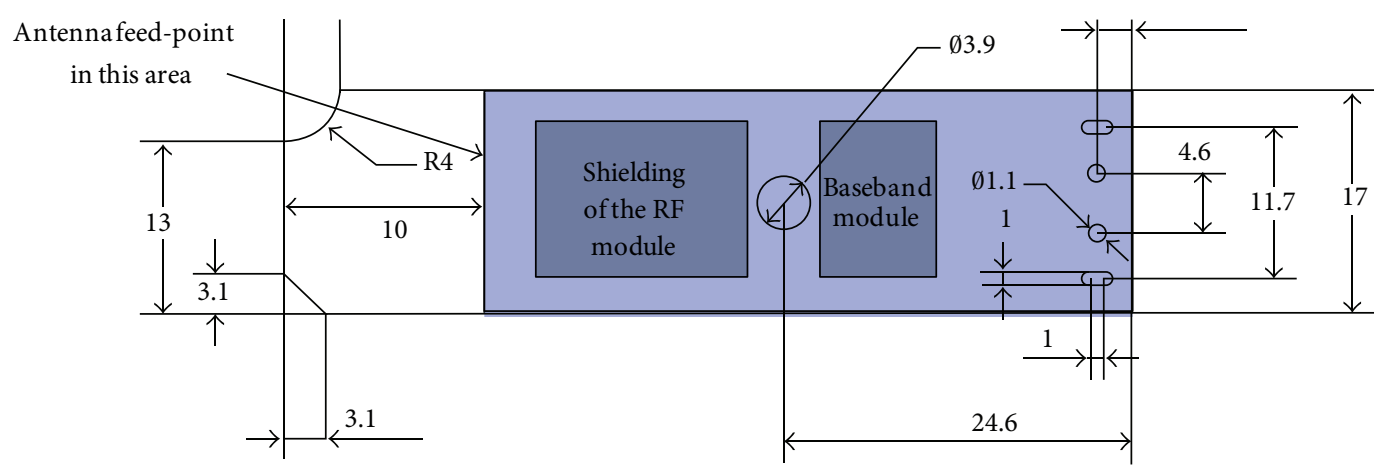

FIgURE 1: PCB layout sketch of the WLAN USB dongle (dimensions in $\mathrm{mm}$ ).

The design of compact antennas benefits from the presence of relatively large ground planes, which ease the constraints on what is usually considered as the radiator itself [16-18]. Yet, when microstrip technology is chosen, the ground plane is expected to be in the back of the PCB. Indeed, most of the known low-cost $\mathrm{WiFi}$ dongle radiator configurations rely on the use of a single-layered PCB with metal on both sides.

In the present scope, this work discusses an IFA-based antenna configuration for a dual-band WLAN USB dongle application. Only a single metal plane PCB was available, thus posing above-the-average constraints to the radiator design. Furthermore, the influences of the dongle case and of the laptop in which the dongle is expected to be plugged in during operation were both considered. As in virtually all related works, the antenna design was numerically assisted by the use of an electromagnetic field simulator. Prototypes were assembled and tested according to the simulated scenarios, measuring performance parameters such as $S_{11}$, radiation efficiency, and power patterns. It is worth mentioning that such antenna was preliminarily assessed in [19], though under a less thorough approach. Relevant gaps left there regarding both the simulation and experimental validation stages are now more properly addressed.

Section 2 addresses the antenna design, highlighting the imposed constraints and the followed method. Simulation results are presented in Section 3, in which the dongle is analyzed both alone and plugged in a typical laptop. Section 4 discusses the experimental validation of the proposed radiator, in terms of both impedance adaptation and radiation performance. Section 5 concludes the paper.

\section{Antenna Design}

2.1. Imposed Constraints. Figure 1 illustrates the layout constraints of the USB dongle. The PCB area available was smaller than $17 \times 60 \mathrm{~mm}^{2}$, from which only the upper $17 \times 10 \mathrm{~mm}^{2}$ side was left for the radiator. Fabrication cost reduction was a priority, in such a way that only one metal layer was made available for both the antenna and all the due electronics, as stated in the introduction. No changes whatsoever were allowed in the area below the space reserved for the radiator. Briefly, the antenna had to be integrated to the PCB using the same single plane and sharing the ground with the electronic circuitry.

The imposed single plane PCB also restrained the antenna choice to a small set of planar configurations. PIFAs and other “2.5D” layouts were thus out of scope.

In this antenna design, the dongle case had to be made of a low-cost injected plastic structure available. However, the electric properties of that material in the WLAN frequency bands were not known beforehand, thus posing an additional issue.

The USB dongle was supposed to operate in both WLAN ISM bands: $2.4-2.5 \mathrm{GHz}$ and $5.15-5.85 \mathrm{GHz}$. It was also expected that the impedance adaptation for a $50-\Omega$ load should provide a reflection coefficient $S_{11}$ no higher than $-10 \mathrm{~dB}$ within the specified bands, though the $-6 \mathrm{~dB}$ limit was acceptable, bearing in mind the natural performance limitations of compact antennas [16-18].

This relative flexibility on the $S_{11}$ performance was allowed also due to the variety of scenarios in which the USB stick was supposed to operate, mainly regarding the laptop influence. Typically, any notebook has many USB ports spread along the keyboard panel sides or at its back, as illustrated in Figure 2. Furthermore, the screen is expected to form an angle around $90^{\circ}$ to $120^{\circ}$ with the keyboard, and such layout may also affect the antenna performance, particularly when the radiator is small, as was the case.

2.2. Methodology. As in most of the other related works, the restricted geometrical specifications imposed led to a numerically assisted design methodology. The suitable electromagnetic field simulator available for this work was Empire XCcel, from IMST [20]. The whole process carried out may be described as follows.

First, taking into account the PCB layout provided in Figure 1, a few basic planar single-band radiator configurations were chosen, based on the available knowledge on compact antennas. In this work, meander line and IFA layouts were tested, simulating such structures directly on the PCB layout provided, with the aid of the field analysis software. Then, a fine tuning of these configurations to the lower frequency band $(2.45 \mathrm{GHz})$ was carried out, from which the IFA-based one presented the best performance, and, for that, 


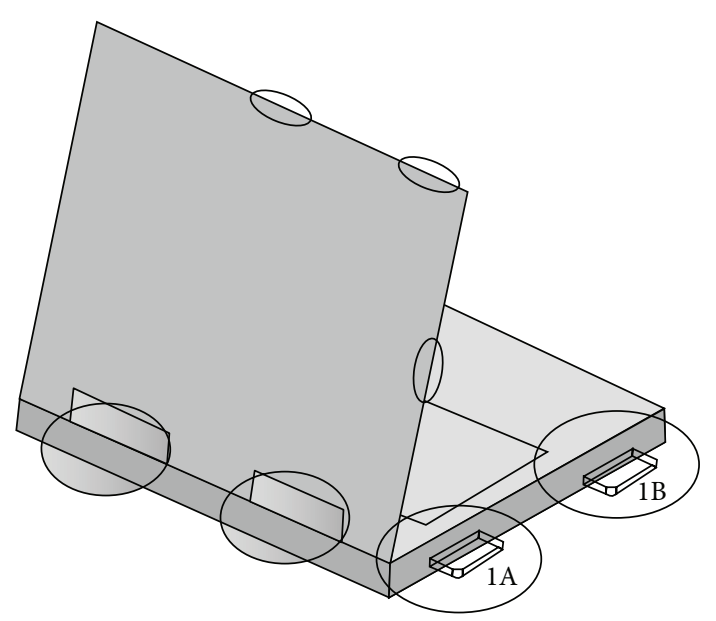

(a)

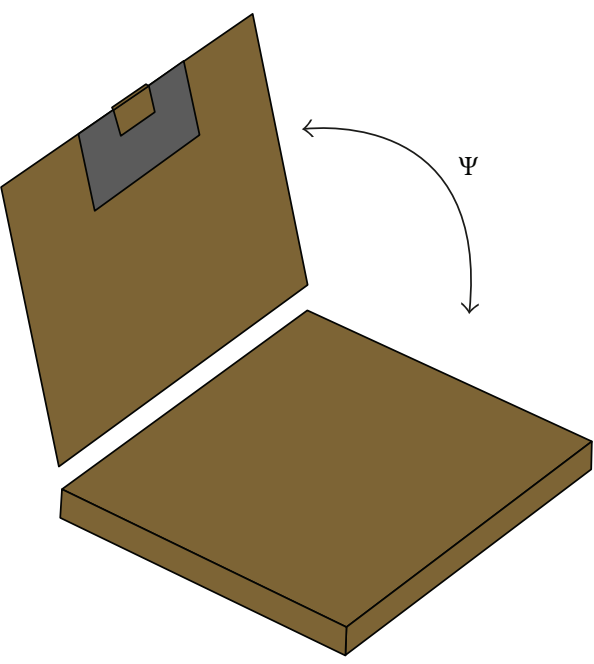

(b)

FIGURE 2: Operation of a WLAN dongle on a laptop and features that potentially affect the antenna performance: (a) USB ports positions and (b) screen-to-base angle $(\Psi)$.

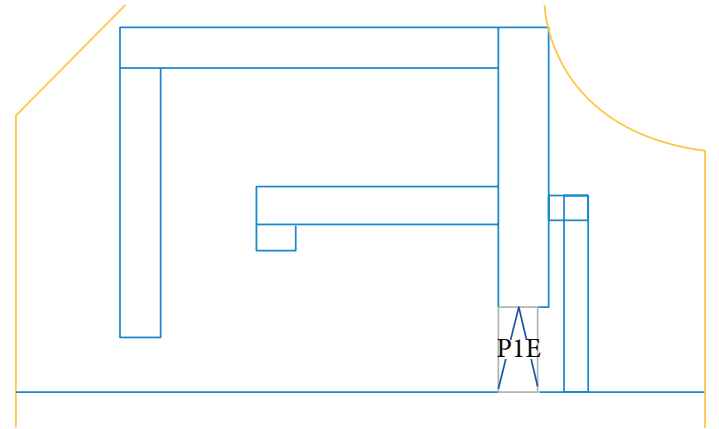

FIGURE 3: Sketch of the radiator configuration proposed as a compact planar dual-band solution (feed gap marked as P1E).

it was selected for prototyping and a preliminary round of measurements.

The need for such initial set of tests was due to the absence of accurate information on the dongle case electrical properties. Therefore, an experimental procedure was set to estimate the resulting frequency shift due to the casing material that would wrap around the PCB. Basically, $S_{11}$ measurements of the prototype $\mathrm{PCB}$ without and with the casing material were performed, from which a $5.4 \%$ downshift (around $130 \mathrm{MHz}$ ) was observed in the operating frequency (resonance or near resonance frequency) of the PCB + case set.

The next step in the design process was the inclusion of another resonance in the upper $5.5 \mathrm{GHz}$ band, while also taking into account the expected frequency shift imposed by the dongle case. It is important to remark that, since no accurate values of the casing material permittivity were available, it was not directly considered in the supporting simulations. What has been done instead was to rescale the antenna to resonate around $5.4 \%$ above the WLAN central frequencies to compensate the lack of the dongle case in the

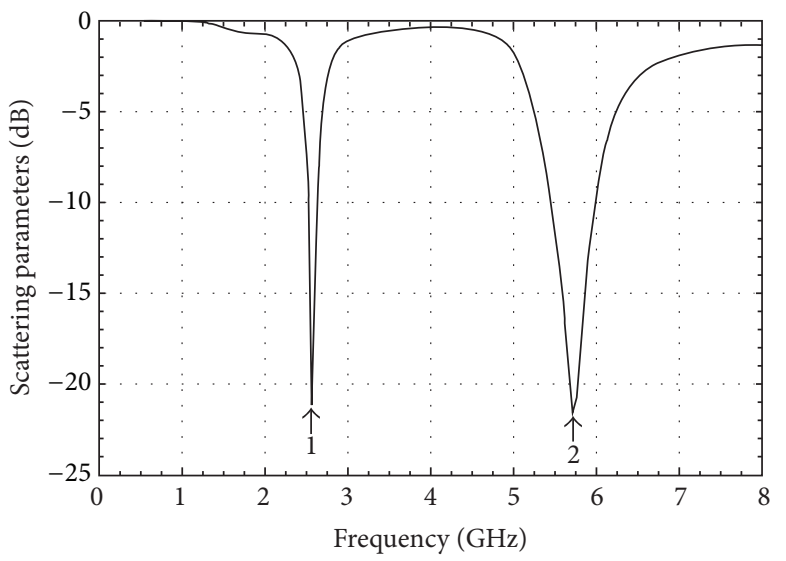

FIGURE 4: $S_{11}$ simulated results of the proposed antenna, when the USB dongle is analyzed alone.

simulation models. Therefore, in this stage of the process, the antenna should be designed to resonate close to $2.58 \mathrm{GHz}$ at the lower band and $5.8 \mathrm{GHz}$ at the upper band.

Since the upper WLAN band was more than an octave above the lower band, a feasible solution could be achieved by the insertion of a second smaller IFA in the same available space, followed by parametrical-based tuning [16-18]. The fine-tuned layout that presented the best performance is sketched in Figure 3. This proposed configuration has a higher frequency branch inserted in the lower frequency IFA (the largest segment) sharing a common return to ground, as well as the feed port. As it can be seen, both the largest and the shortest segments are actually not " $F$ " shaped, since both had to be round bent at their ends in order to make the whole set fit to the restricted available area. It is also worth mentioning that, from what could be observed during the 


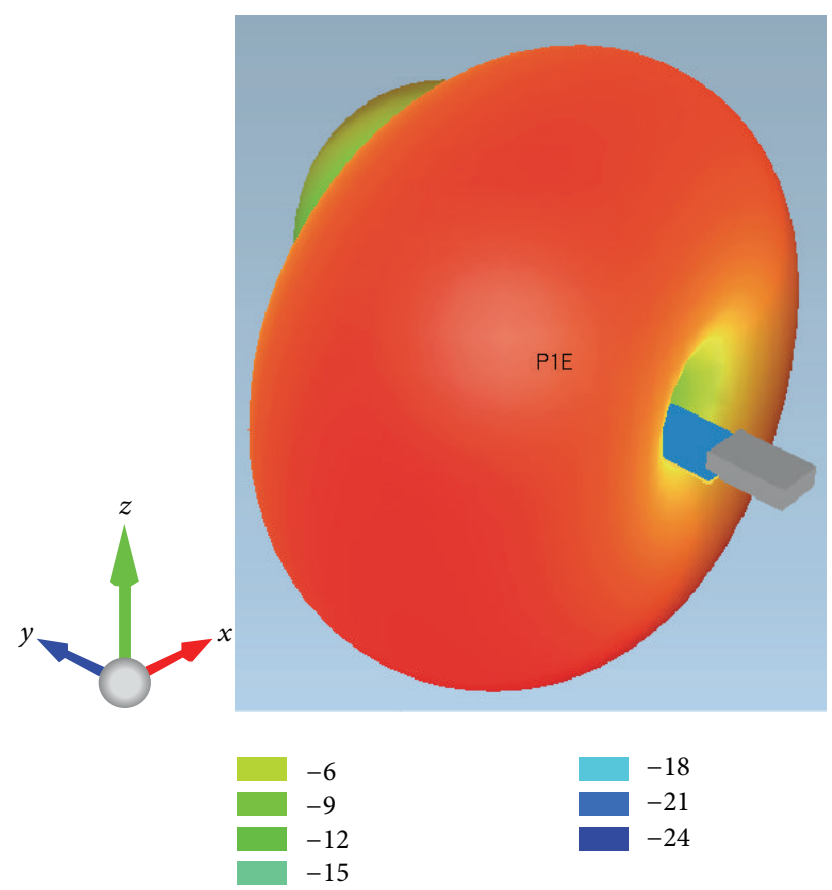

FIGURE 5: Simulated 3D radiation pattern of the proposed antenna, when the USB dongle is analyzed alone, at $2.56 \mathrm{GHz}$.

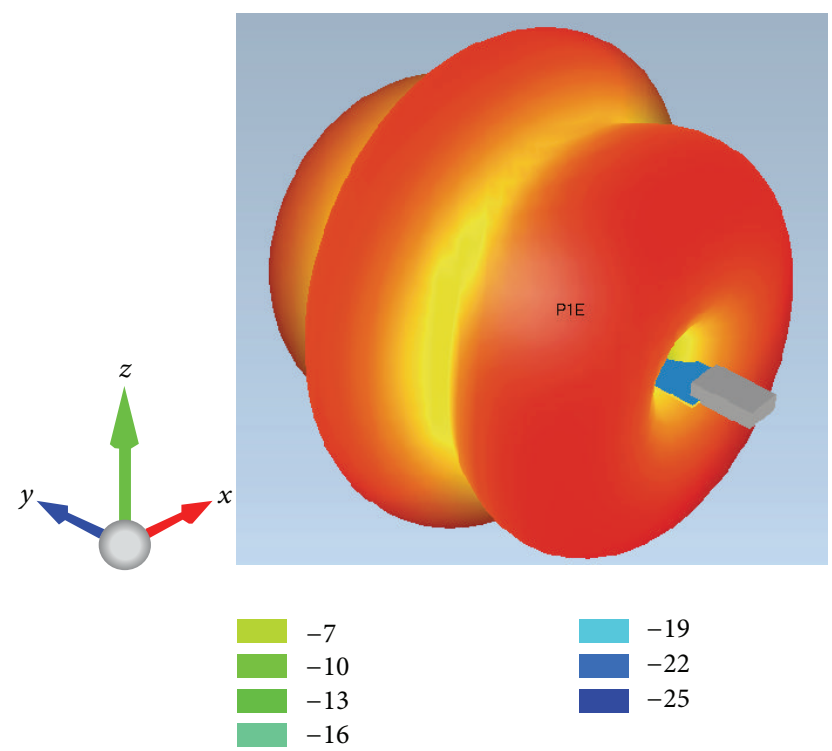

FIGURE 6: Simulated 3D radiation pattern of the proposed antenna, when the USB dongle is analyzed alone, at $5.72 \mathrm{GHz}$.

tuning simulations, the parameters that affected most critically the $S_{11}$ performance were the feed gap width and the total length of each branch.

The radiator configuration in Figure 3 was actually achieved after another set of tuning simulations needed in order to evaluate the effect of the laptop on the antenna performance. At this level, the simulations took into account the four relative USB ports indicated in Figure 2, with the laptop screen angle $\Psi=90^{\circ}$. The basic parameter observed

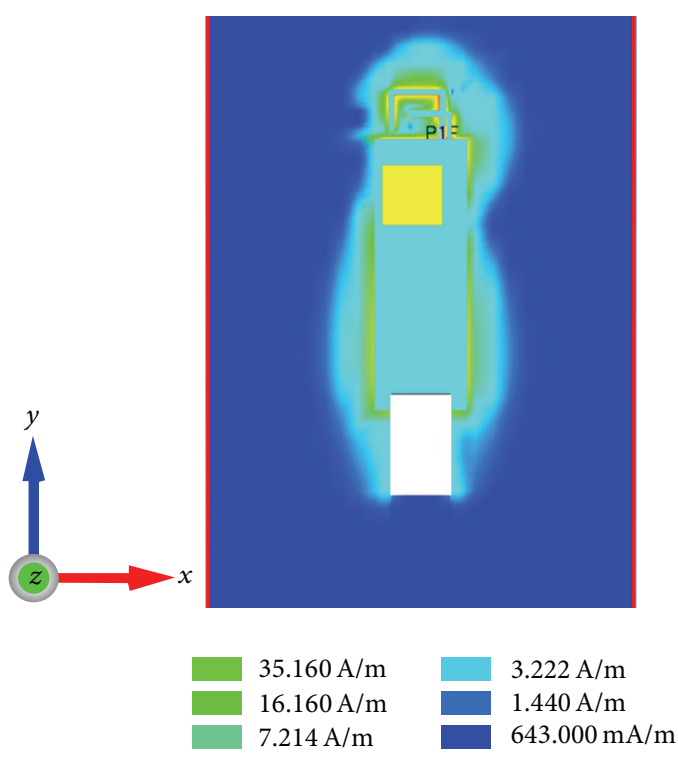

FIGURE 7: Simulated current density distribution of the proposed antenna, when the USB dongle is analyzed alone, at $2.56 \mathrm{GHz}$.

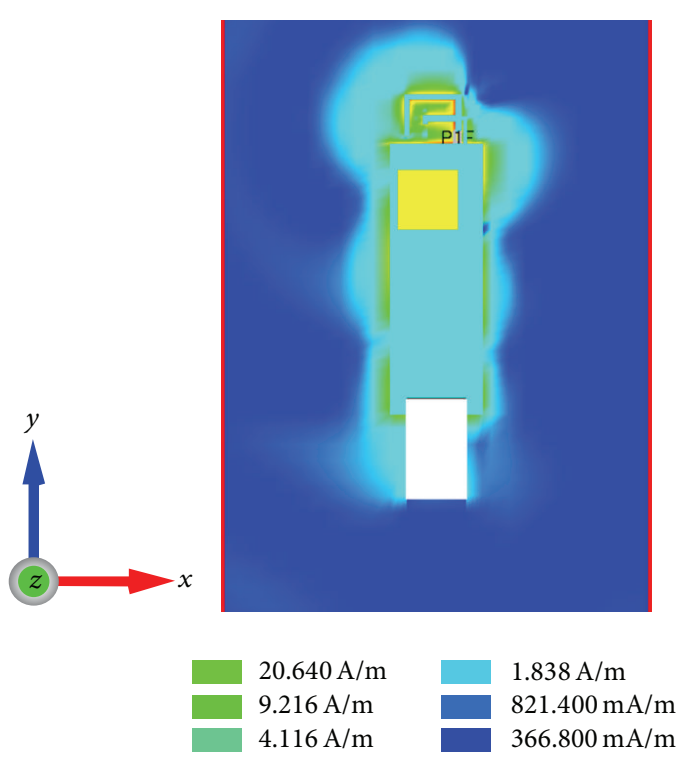

FIGURE 8: Simulated current density distribution of the proposed antenna, when the USB dongle is analyzed alone, at $5.72 \mathrm{GHz}$.

to guide this final tuning round was $S_{11}$, since typically there is not much that can be done regarding the pattern of compact antennas [16-18]. Anyway, the radiation pattern behavior should be observed, with and without the influence of the laptop, in order to see how omnidirectional the dongle can be.

Finally, the simulated layout that provided the best overall performance was picked for prototyping and qualification tests. These trials should include $S_{11}$ measurements of the antenna impedance in a number of situations, particularly with the dongle plugged in different USB ports of a typical laptop, with its screen opened, as illustrated in Figure 2. With 


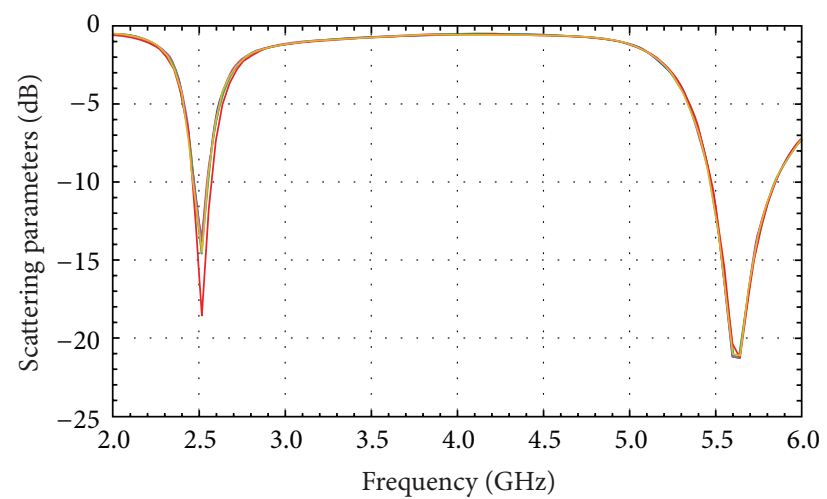

(a)

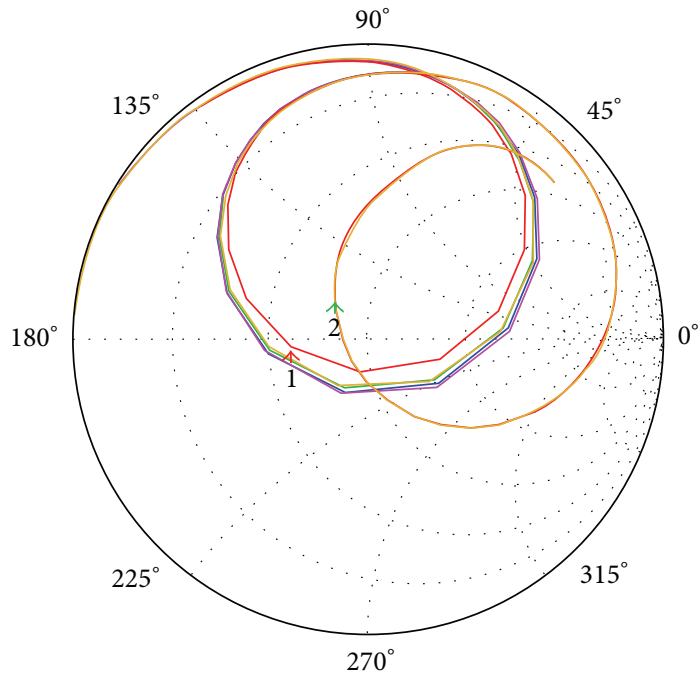

(b)

FIGURE 9: Simulated (a) $S_{11}$ and (b) Smith Chart of the proposed antenna, with the influence of the laptop (parameter sweep of the insertion point, in the backside position).

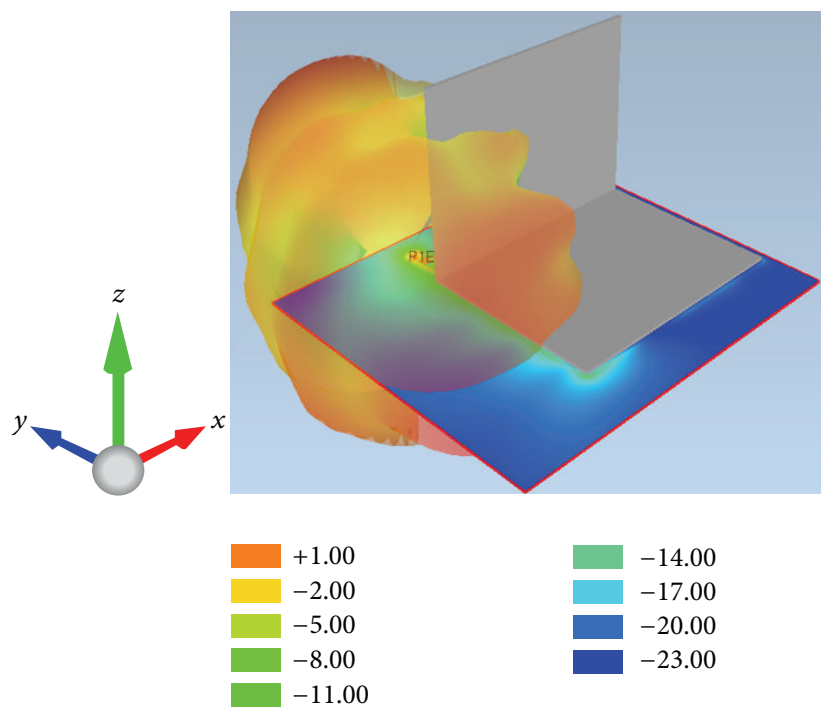

FIGURE 10: Simulated 3D radiation pattern of the proposed antenna, at $2.56 \mathrm{GHz}$, with the influence of the laptop.

proper lab facilities available such as an anechoic chamber, it is also desirable to measure radiation patterns and efficiency. In this work, the validation tests comprised both the impedance adaptation and radiation performance aspects, in realistic scenarios (dongle set assembled with its plastic casing and plugged in a laptop) as addressed in Section 4.

\section{Simulation Results}

In this section, the simulated results of the best achieved configuration, sketched in Figure 3, are presented. The discussion includes not only the antenna behavior on the dongle alone, but also the influence of a laptop, which corresponds to the more realistic operation scenario.
3.1. Dongle PCB Alone. Figure 4 shows the $S_{11}$ simulation results of the proposed antenna. A dual-band operation was achieved, with the best impedance matching frequencies at 2.56 and $5.72 \mathrm{GHz}$. As addressed in Section 2, since the dongle case was not directly taken into account in the simulator, the target center frequencies of the lower and higher operation bands were 2.58 and $5.8 \mathrm{GHz}$, respectively. At this stage of the design process and bearing in mind that the laptop effect would be evaluated, these frequency values were acceptable enough.

Figures 5 and 6 show the simulated radiation patterns of the dongle alone, without casing, and basically a dipole-like behavior is seen, as expected. The compact antenna limitation to radiate only the lowest order spherical modes is clearer in Figure 5, where the pattern is quasi-omnidirectional, given 
that the radiator largest branch length $(24 \mathrm{~mm})$ is around five times shorter than the wavelength of the lower band $(117 \mathrm{~mm}$ at $2.56 \mathrm{GHz}$ ) [16-18].

Electromagnetic field simulators such as the one used in this work also provide interesting information on the current density distribution. Important insights into the current paths may be drawn from the analysis of this parameter that may be helpful to retune the antenna, during the design phase. Figures 7 and 8 present the simulated distributions of the dongle alone at 2.56 and $5.72 \mathrm{GHz}$, respectively.

Observing the current density distributions at each band, the smaller branch of the antenna is not so active in the lower band; see Figure 7. Also, the distribution along the PCB edges is almost uniform. On the other hand, at the higher band, the small branch is active, while the current distribution along the edges of the PCB is not uniform anymore, as seen in Figure 8. Thus, the radiation behavior seems to be the combination of a first order mode associated with the shorter tip-bent "F" branch with a second order mode related to the longer one. The sidelobes on the pattern of Figure 6 corroborate that.

3.2. Laptop Influence. The laptop was modeled as an open $90^{\circ}$ wedge of perfect electric conductor (PEC) planes. Simulations were performed considering the dongle inserted in the back of or alongside the laptop, in different plug-in positions. The worst case in terms of detuning of the $S_{11}$ response achieved in the dongle alone scenario was observed on the backside insertion configuration.

Figure 9 shows the $S_{11}$ results of a parameter sweep of the backside plug-in scenario, that is, testing different plugin relative positions along the back of the laptop. Even in this worst-case scenario, the sensibility of the tuning to the insertion position was relatively low in terms of frequency shift, as it can be seen. This may be interpreted as a consequence of an overcritically matching coupling condition [21], achieved with the present design, observed on the Smith Chart of Figure 9.

A more physical argument for such frequency stability is related to the current density distribution on the ground. As discussed in [9], when the current density on the ground plane is low enough compared to the one present on the main radiator element, the sensibility of the operation frequency to the laptop is expected to be low. Therefore, by plugging the dongle in the laptop, the antenna ground is expanded (PCB + laptop), reducing even more its already relatively low current density.

Figures 10 and 11 illustrate the radiation patterns of the dongle plugged in the backside of the laptop, at 2.56 and $5.72 \mathrm{GHz}$, respectively. As already mentioned in the previous section, the design was driven mostly by the impedance adaptation. Yet, with the low-cost constraint in hands, there has been no attempt to jointly tune the antenna considering the $S_{11}$ and the radiation performances. Anyway, the results are acceptable, in the sense that enough radiated energy is available in all directions, even in the worst case of the dongle plugged in the back.

It is worth remarking that the ground density current argument that fits well for understanding the impedance

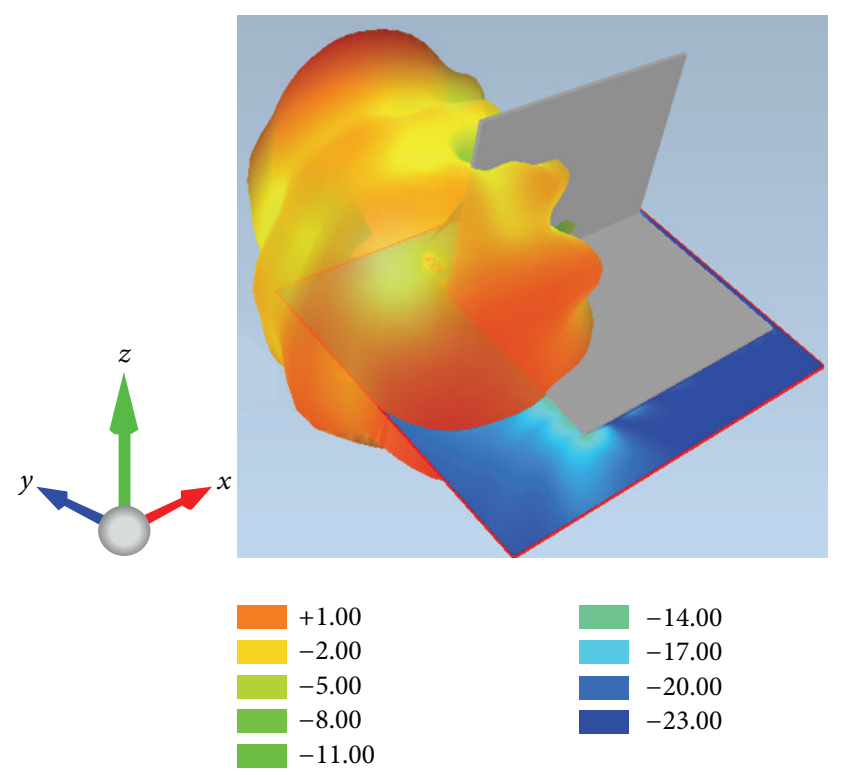

FIGURE 11: Simulated 3D radiation pattern of the proposed antenna, at $5.72 \mathrm{GHz}$, with the influence of the laptop.

matching stability does not apply as much for the radiation behavior. The coupling between the main radiator and the screen of the laptop induces additional currents on this surface which is almost orthogonal to the $\mathrm{PCB}$, contributing to the deformation of the radiation patterns as seen in Figures 10 and 11. Nevertheless, bearing in mind that these simulations considered a PEC laptop model, it is reasonable to expect lower coupling in the actual application.

\section{Experimental Qualification}

4.1. $S_{11}$ Measurements. A prototype of the best antenna layout achieved, shown in Figure 3, was assembled. It emulates the dongle, yet without the embedded electronics. Figure 12 shows a few snapshots of the assembled set. In order to measure the antenna parameters, external access to the feed port was needed. For that, the small piece of $50-\Omega$ coaxial cable seen in Figure 12 was used. While the cable end external to the dongle was terminated by an SMA connector, the opposite end was soldered to the feed gap marked as P1E in Figure 3. $S_{11}$ measurements of the antenna either without or with the plastic case were performed. The effect of the laptop was assessed as well.

Figure 13 shows the measurement results of 4 different relative plug-in configurations. The snapshot of the $S_{11}$ measurement setup using a laptop, with the USB dongle plugged in the backside, and with the screen opened at a $90^{\circ}$ angle is also shown. The plotted curves present a good frequency stability behavior within the $-10 \mathrm{~dB}$ threshold in most cases and within $-6 \mathrm{~dB}$ for all cases. This result is coherent with the overcritical impedance adaptation approach pursued in the simulations, as well as the ground current density argument, both addressed in Section 3.

It can also be noticed in Figure 13 that the impedance adaptation in the lower band was poorer than that in the 


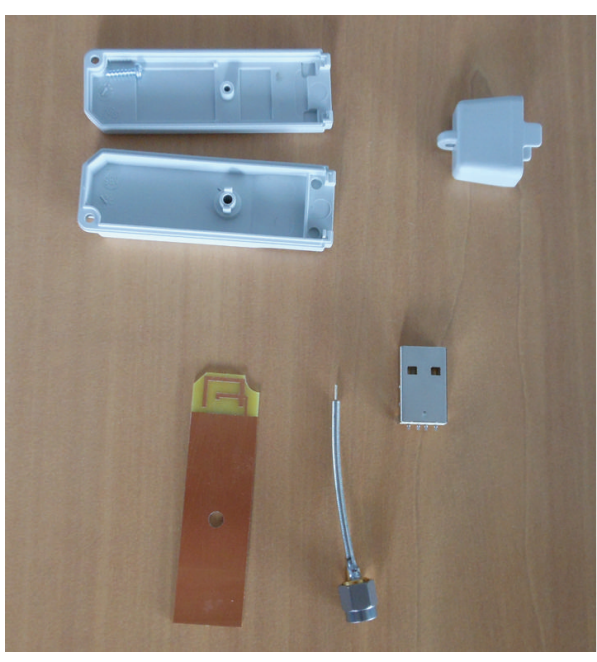

(a)

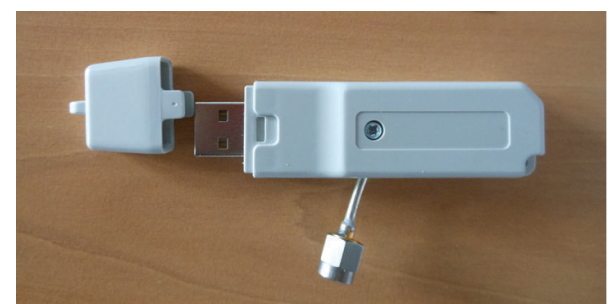

(b)

FIGURE 12: Dual-band antenna prototype: (a) dongle set and (b) dongle assembled for tests.

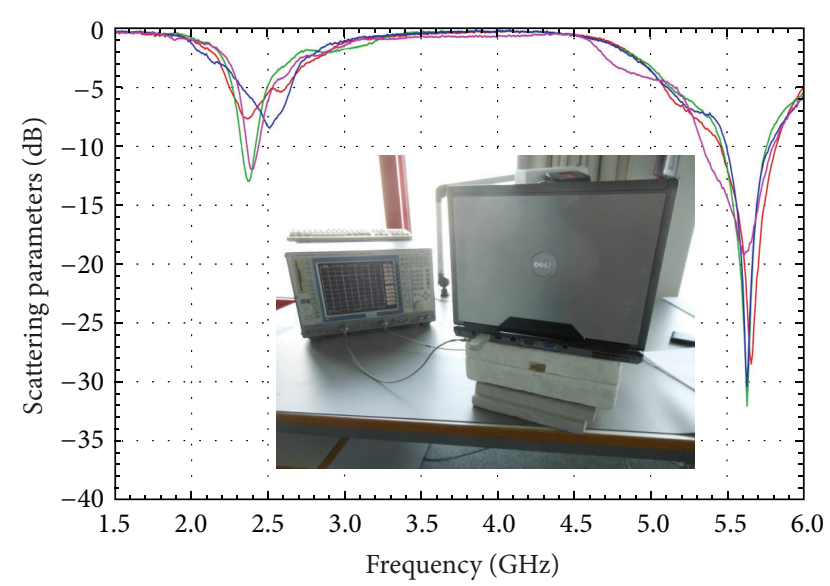

Figure 13: Experimental assessment of the laptop effect on the antenna performance: measured $S_{11}$ curves corresponding to 4 different plug-in positions alongside the computer base and a snapshot of the measurement setup.

upper band, while the simulated results in Section 3 predicted a more balanced performance, with both $S_{11}$ notches close to $-20 \mathrm{~dB}$. This difference is mostly due to the small antenna behavior present in the lower band. As reported in the literature [17], measuring small antenna parameters is usually a challenging task. The list of issues includes low radiation resistance, high input reactance, high losses, and the balancing state of the antenna input regarding the presence of a poor ground reference, among others. Therefore, the measurement uncertainty in the lower band is expected to be higher than in the upper band, giving rise to differences such as the one remarked.

4.2. Radiation Pattern and Efficiency Measurements. Figures 14 and 15 show $2 \mathrm{D}$ cuts of the measured gain patterns of the proposed antenna at 2.45 and $5.5 \mathrm{GHz}$, respectively. Gain is shown in its partial components on directions $\theta$ and $\phi$, in
TABLE 1: Measured gain $(G)$ and efficiency $(\eta)$ of the proposed antenna, when the dongle is plugged in the back of a laptop, at different frequencies $(f)$.

\begin{tabular}{lcccccc}
\hline$f(\mathrm{GHz})$ & 2.35 & 2.45 & 2.55 & 5.40 & 5.50 & 5.60 \\
\hline$G(\mathrm{dBi})$ & 2.6 & 2.8 & 2.8 & 5.7 & 5.9 & 6.8 \\
\hline$\eta(\%)$ & 45.0 & 59.0 & 60.9 & 72.8 & 75.2 & 79.3 \\
\hline
\end{tabular}

order to provide information on the antenna polarization. These measurements were taken with the dongle plugged in the back of an opened laptop $\left(\Psi=90^{\circ}\right)$. The erratic polarization behavior observed was not an issue, because, typically, for this kind of application, there is no design constraint in this regard. Most importantly, the quasi-omnidirectional aspect expected from simulations was confirmed, as seen at both frequency bands. Measurements were carried out also at other frequencies within the WLAN operation bands, and similar general behavior was observed.

Table 1 brings detailed information on other important radiation parameters obtained from measurements: maximum gain and total efficiency. The lower band presents the worst results, as expected, since the radiator is compact compared to the wavelength. Nevertheless, the gain is relatively high for such scenario (close to $3 \mathrm{~dB}$ ), and even the efficiency achieved is above $45 \%$, a reference value typically considered for small antennas $[16,17]$. The higher band is less critical regarding the antenna size, and for that both gain and efficiency are high, around $6 \mathrm{~dB}$ and $75 \%$, respectively, with values coherent with those expected from monopolelike radiators.

Still concerning the upper band, it is worth noting that the design goal for its center frequency was $5.5 \mathrm{GHz}$, but the assembled antenna presented its second autoresonance close to $5.6 \mathrm{GHz}$, as seen in Figure 13. Thus, the highest gain at $5.6 \mathrm{GHz}$, almost $1 \mathrm{~dB}$ above the gain at $5.5 \mathrm{GHz}$, was actually a coherent, rather than unexpected, behavior. 


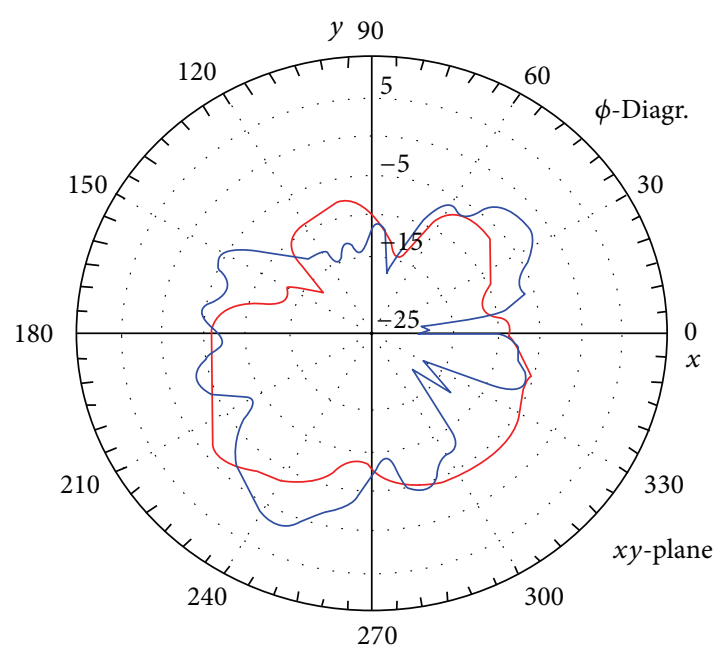

(a)

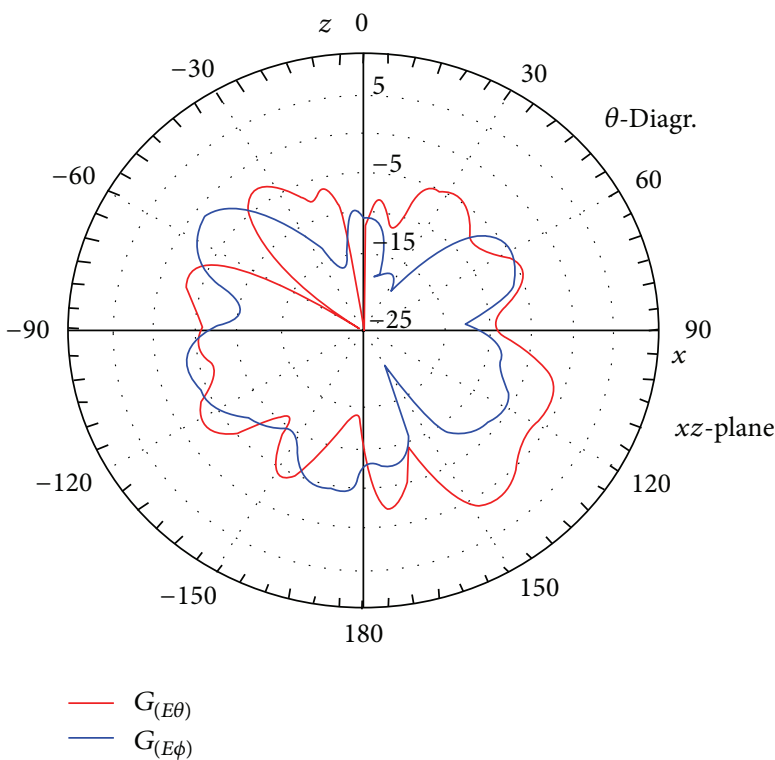

(c)

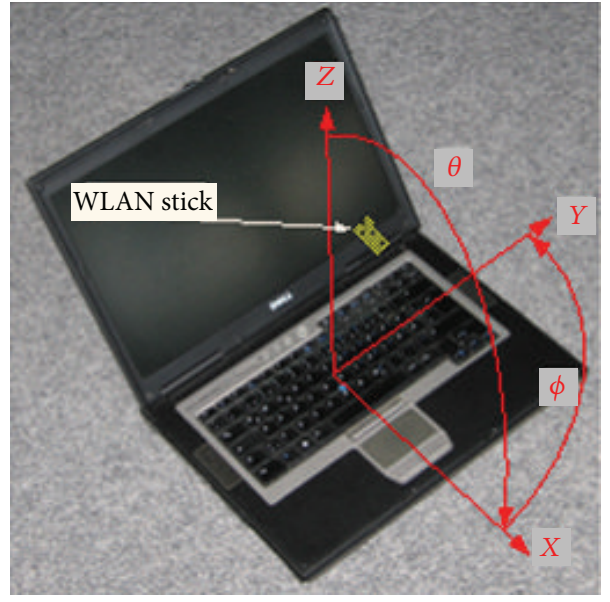

(b)

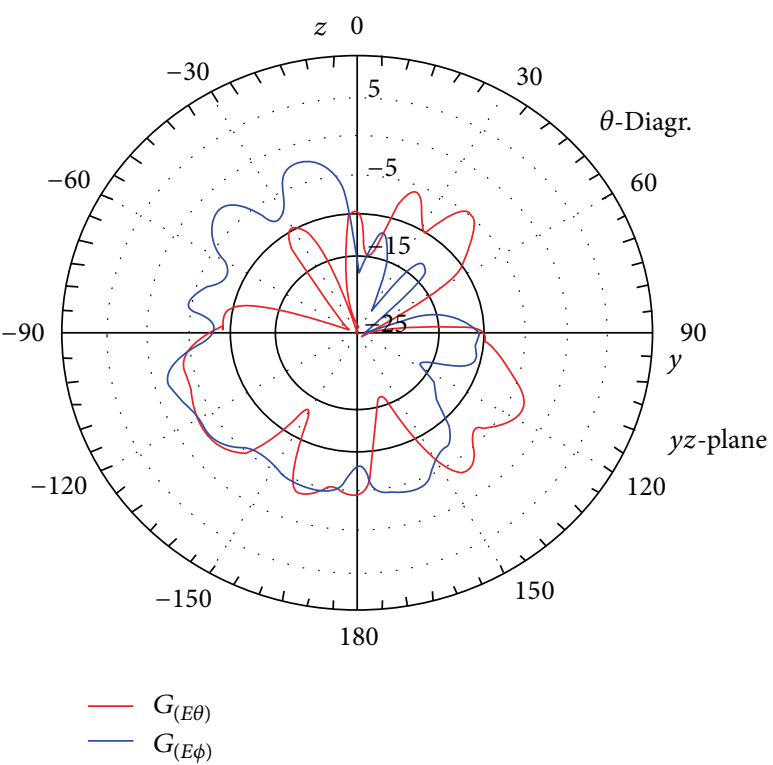

(d)

FIGURE 14: Measured gain pattern of the proposed antenna, when the dongle is plugged in the back of a laptop, at $2.45 \mathrm{GHz}$.

\section{Concluding Remarks}

In this paper, the design of a novel compact dual-band planar antenna for a low-cost USB WLAN dongle was discussed. Among the main constraints, the radiator element had to fit within a space as small as $17 \times 10 \mathrm{~mm}^{2}$. Furthermore, the planar feature was actually imposed, since the antenna should be integrated to the device PCB, which had nothing but a single metal layer available. As a result, the set of pertinent basic antenna configuration choices at the beginning of the design process was more limited than usual. An IFA-based shape was the one that gave the best results, with the final shape actually resembling a tip-bent "F", since the largest branch of the radiator had to be bent to fit within the available area.
As in most of the other related works, a numerically assisted methodology was chosen for the antenna design. The electromagnetic field simulator available for this work was the software Empire XCcel. The process should include the effects of the plastic casing of the dongle, as well as the influence of a typical laptop in use, in which the dongle was supposed to be plugged in during operation.

While the laptop effect could be analyzed directly using the software, the lack of accurate information on the dongle case material electrical properties did not allow its inclusion in the simulations. The adopted solution came as an intermediate experimental step, in which a preliminary prototype tuned to the $2.45 \mathrm{GHz}$ band was assembled and measured with and without the case. From this procedure, the frequency shift on the $S_{11}$ response could have been estimated, 


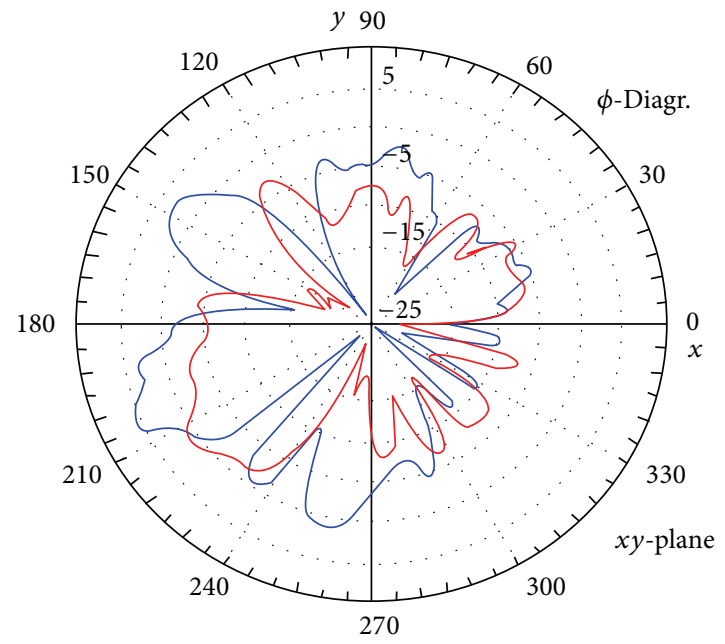

(a)

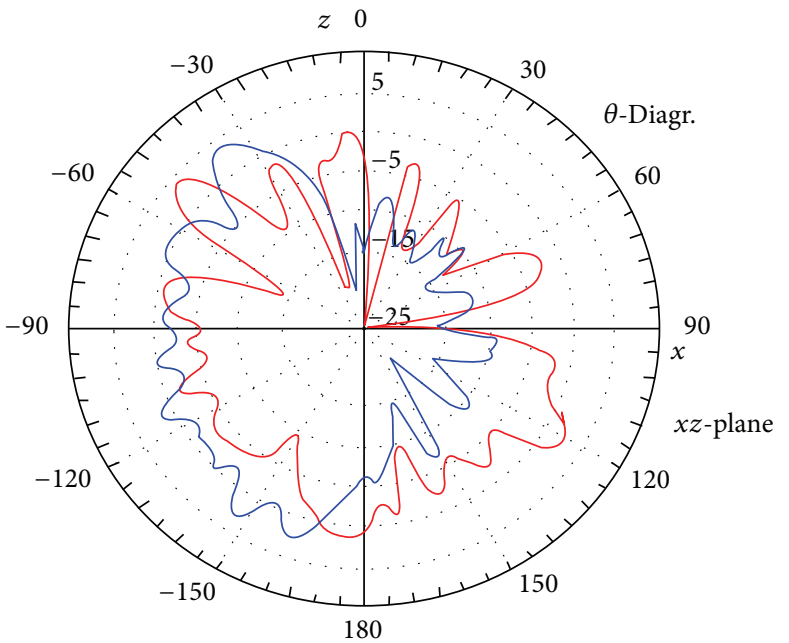

$-G_{(E \theta)}$

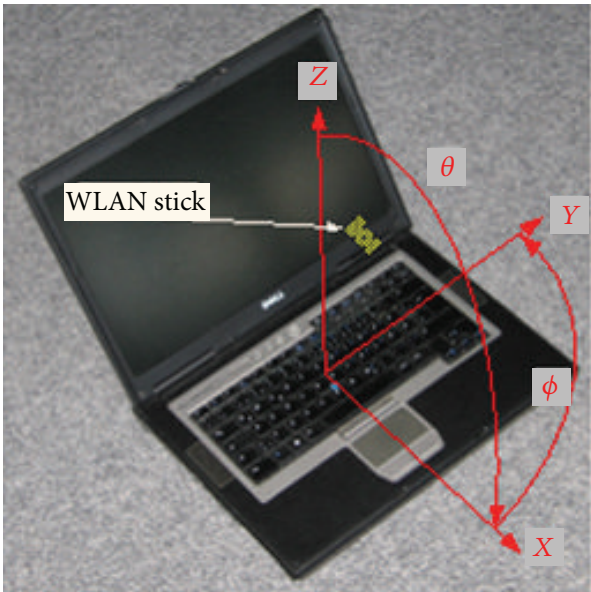

(b)

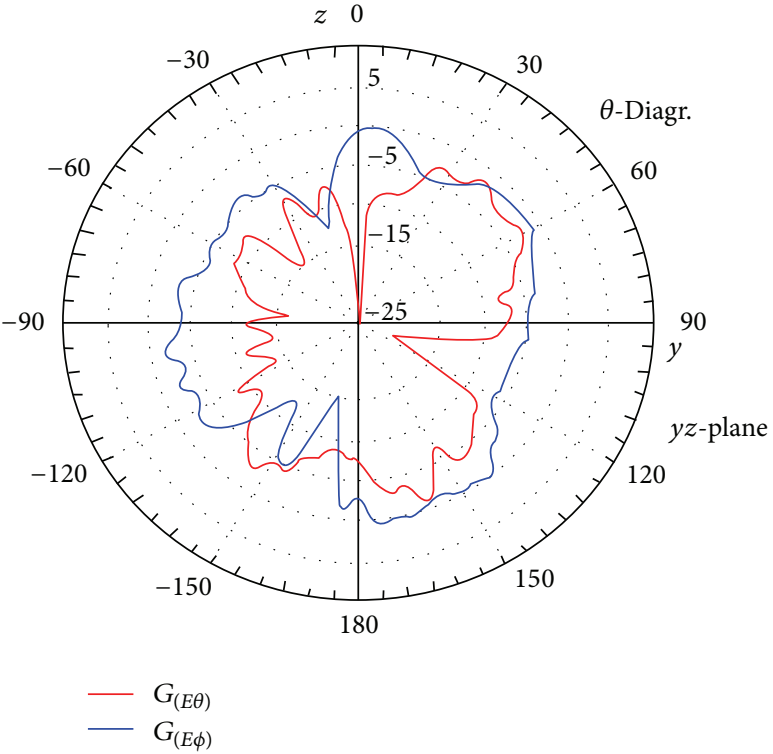

(d)

FIGURE 15: Measured gain pattern of the proposed antenna, when the dongle is plugged in the back of a laptop, at $5.5 \mathrm{GHz}$.

and, based on that, higher design target frequencies were considered on the simulations, to compensate the effect of the plastic casing.

After the final tuning step of the design process, in which the $S_{11}$ response was taken as the main performance parameter to be observed, a prototype of the best configuration achieved was assembled. Measurements were carried out to validate the proposed design. The impedance matching performance of the prototype was coherent with the simulated results, presenting a desirable frequency stability regarding the laptop influence. That is, no matter the position where the dongle was plugged in, the $S_{11}$ bandwidth was kept within acceptable limits.

The radiation behavior was also assessed by measurements. Power patterns kept the quasi-omnidirectional shape expected from simulations and from theory. Gain and efficiency were also measured, with good results for such a kind of compact radiator. Overall, the proposed IFA-based antenna proved to be a suitable choice for low-cost compact WLAN dongle devices.

\section{Conflict of Interests}

The authors declare that there is no conflict of interests regarding the publication of this paper.

\section{Acknowledgments}

This work was supported in part by the Brazilian Council for Scientific and Technological Development (CNPq) under 
Grant 306819/2012-0 and the Rio de Janeiro Foundation for Research Support (FAPERJ) under Project E-26/101497/2010. The authors would also like to express their gratitude to the European School of Antennas lecturers and technical staff from IMST-GmbH who managed the Industrial Antenna Design Course in April 2013, from which most of the present contribution was derived.

\section{References}

[1] Y. Xiong and Z. Qu, "Antenna 3D Pad printing solution evaluation," in Proceedings of the International Symposium on Antennas and Propagation (APSURSI '11), pp. 2773-2776, Spokane, Wash, USA, July 2011.

[2] M. K. Hosain, A. Kouzani, and S. Tye, "Multi-layer implantable antenna for closed loop deep brain stimulation system," in Proceedings of the International Symposium on Communications and Information Technologies (ISCIT '12), pp. 643-648, Outrigger Surfers Paradise, October 2012.

[3] C. B. Ravipati, D. R. Jackson, and H. Xu, "Center-fed microstrip antennas with shorting vias for miniaturization," in Proceedings of the Antennas and Propagation Society International Symposium and USNC/URSI Meeting, pp. 281-284, July 2005.

[4] J. Kruys and L. Qian, RF Spectrum With Commodity Wireless Technologies: Theory and Practice, Springer, 2011.

[5] W.-S. Chen, W.-C. Jhang, and H.-D. Chen, "Small and compact MIMO antenna for USB dongle applications," in Proceedings of the Cross Strait Quad-Regional Radio Science and Wireless Technology Conference (CSQRWC '11), pp. 438-441, Harbin, China, July 2011.

[6] Q. Luo, C. Quigley, J. R. Pereira, and H. M. Salgado, "Inverted-L antennas array in a wireless USB dongle for MIMO application," in Proceedings of the 6th European Conference on Antennas and Propagation (EUCAP '12), pp. 1909-1912, Prague, Czech Republic, March 2012.

[7] V. Ssorin, A. Artemenko, A. Maltsev, A. Sevastyanov, and R. Maslennikov, "Compact MIMO microstrip antennas for USB dongle operating in $2.5-2.7 \mathrm{GHz}$ frequency band," International Journal of Antennas and Propagation, vol. 2012, Article ID 793098, 12 pages, 2012.

[8] M. Han and J. Choi, "Compact multiband MIMO antenna for next generation USB dongle applications," Microwave and Optical Technology Letters, vol. 54, no. 1, pp. 246-250, 2012.

[9] J. Ou Yang, J. Zhang, K. Z. Zhang, and F. Yang, "Compact folded dual-band slot antenna for wireless communication USB dongle application," Journal of Electromagnetic Waves and Applications, vol. 25, no. 8-9, pp. 1221-1230, 2011.

[10] W.-C. Su and K.-L. Wong, "Internal PIFAs for UMTS/WLAN/ WiMAX multi-network operation for a USB dongle," Microwave and Optical Technology Letters, vol. 48, no. 11, pp. 22492253, 2006.

[11] Y. Yu and J. Choi, "Compact internal inverted-F antenna for USB dongle applications," Electronics Letters, vol. 45, no. 2, pp. 92-94, 2009.

[12] S.-H. Lee and Y. Sung, "Multiband antenna for wireless USB dongle applications," IEEE Antennas and Wireless Propagation Letters, vol. 10, pp. 25-28, 2011.

[13] W.-C. Liu and Y.-L. Chen, "Compact strip-monopole antenna for WLAN-band USB dongle application," Electronics Letters, vol. 47, no. 8, pp. 479-480, 2011.
[14] Q. Luo, H. M. Salgado, and J. R. Pereira, "Compact printed Cshaped monopole antenna with chip inductor," in Proceedings of the International Symposium on Antennas and Propagation (APSURSI '11), pp. 156-159, Spokane, Wash, USA, July 2011.

[15] S. Chaimool, C. Chokchai, and P. Akkaraekthalin, "Triple-band USB dongle antenna using fractal and shorted loops for WiFi and WiMAX applications," Microwave and Optical Technology Letters, vol. 55, no. 4, pp. 863-866, 2013.

[16] J. Volakis, C. C. Chen, and K. Fujimoto, Small Antennas: Miniaturization Techniques and Applications, McGraw-Hill, 2010.

[17] L. Jofre, M. Martinez-Vazquez, R. Serrano, and G. Roqueta, Handbook on Small Antennas, EurAAP, 2012.

[18] J. Anguera, A. Andújar, M. C. Huynh, C. Orlenius, C. Picher, and C. Puente, "Advances in antenna technology for wireless handheld devices," International Journal of Antennas and Propagation, vol. 2013, Article ID 838364, 25 pages, 2013.

[19] B. R. Franciscatto, H. Adel, M. H. C. Dias, and T. P. Vuong, "A compact IFA-based dual-band planar antenna for WiFi USB dongles," in Proceedings of the 8th European Conference on Antennas and Propagation (EuCAP '14), pp. 1-5, The Hague, The Netherlands, April 2014.

[20] http://www.empire.de/.

[21] J. Rahola, "Bandwidth potential and electromagnetic isolation: tools for analysing the impedance behaviour of antenna systems," in Proceedings of the 3rd European Conference on Antennas and Propagation (EuCAP '09), pp. 944-948, Berlin, Germany, March 2009. 

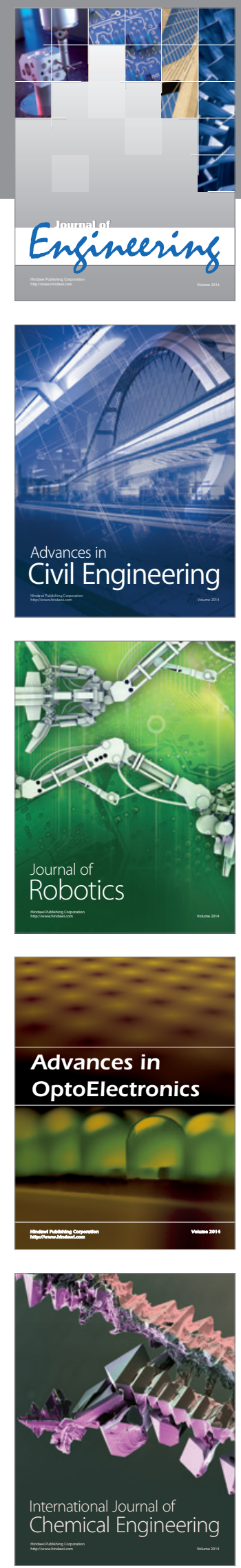

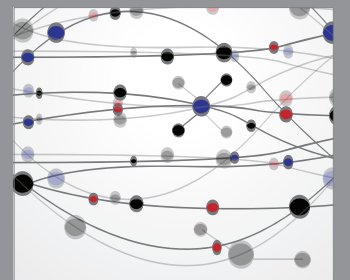

The Scientific World Journal
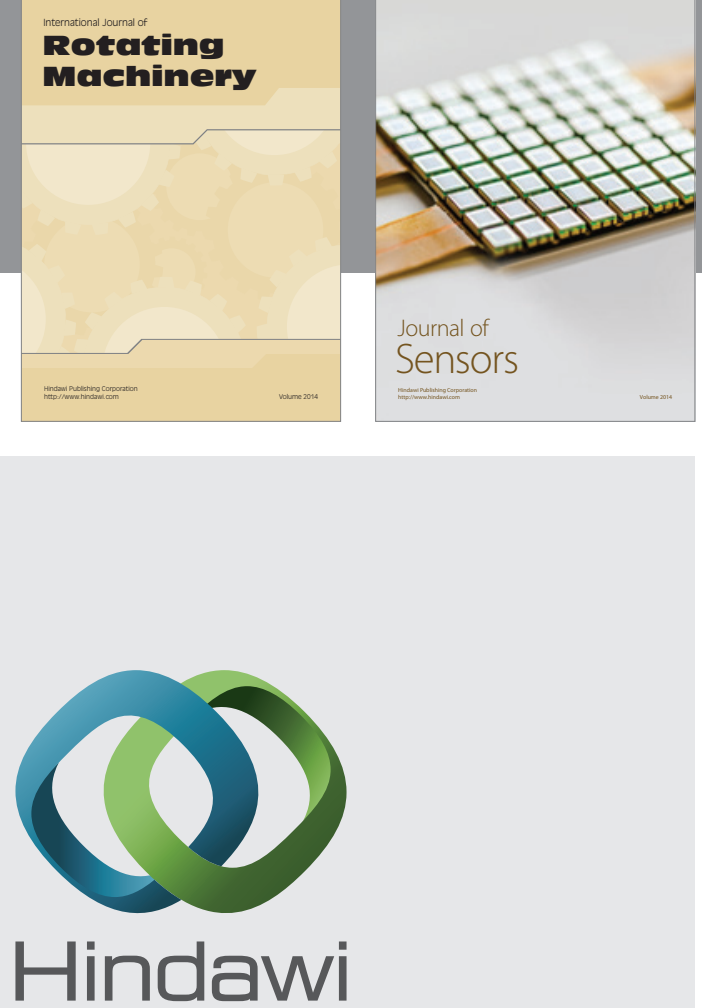

Submit your manuscripts at http://www.hindawi.com
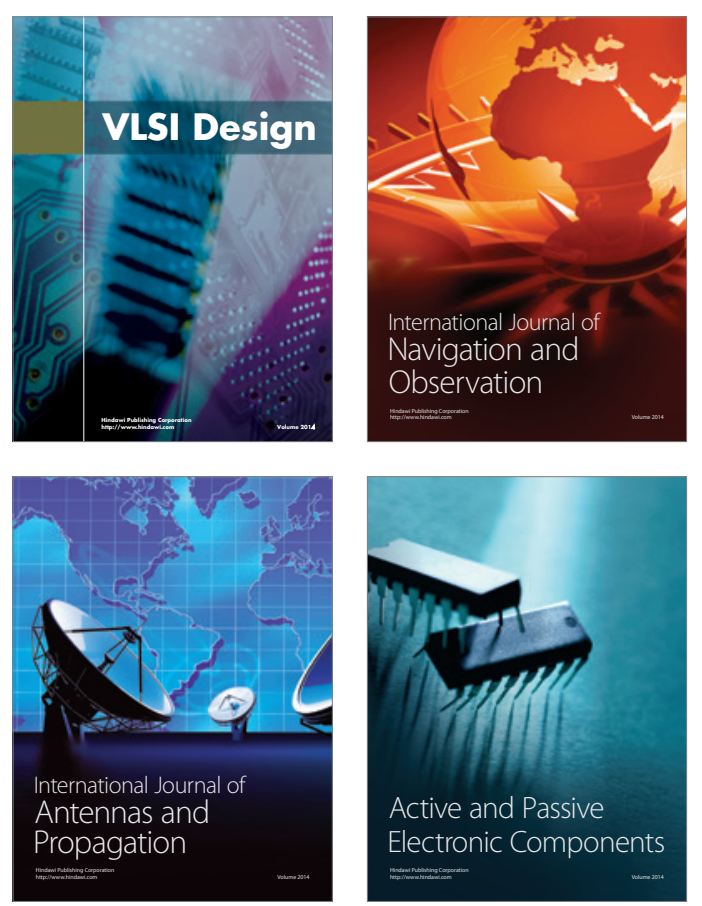
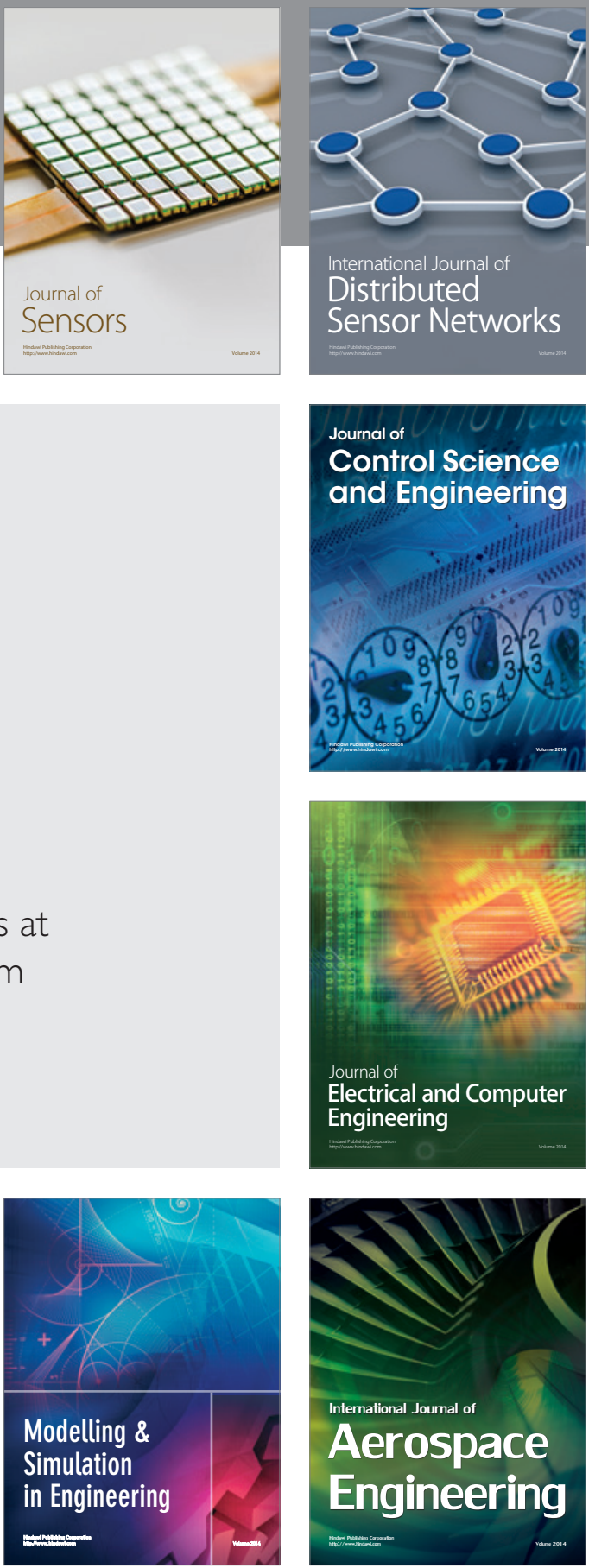

Journal of

Control Science

and Engineering
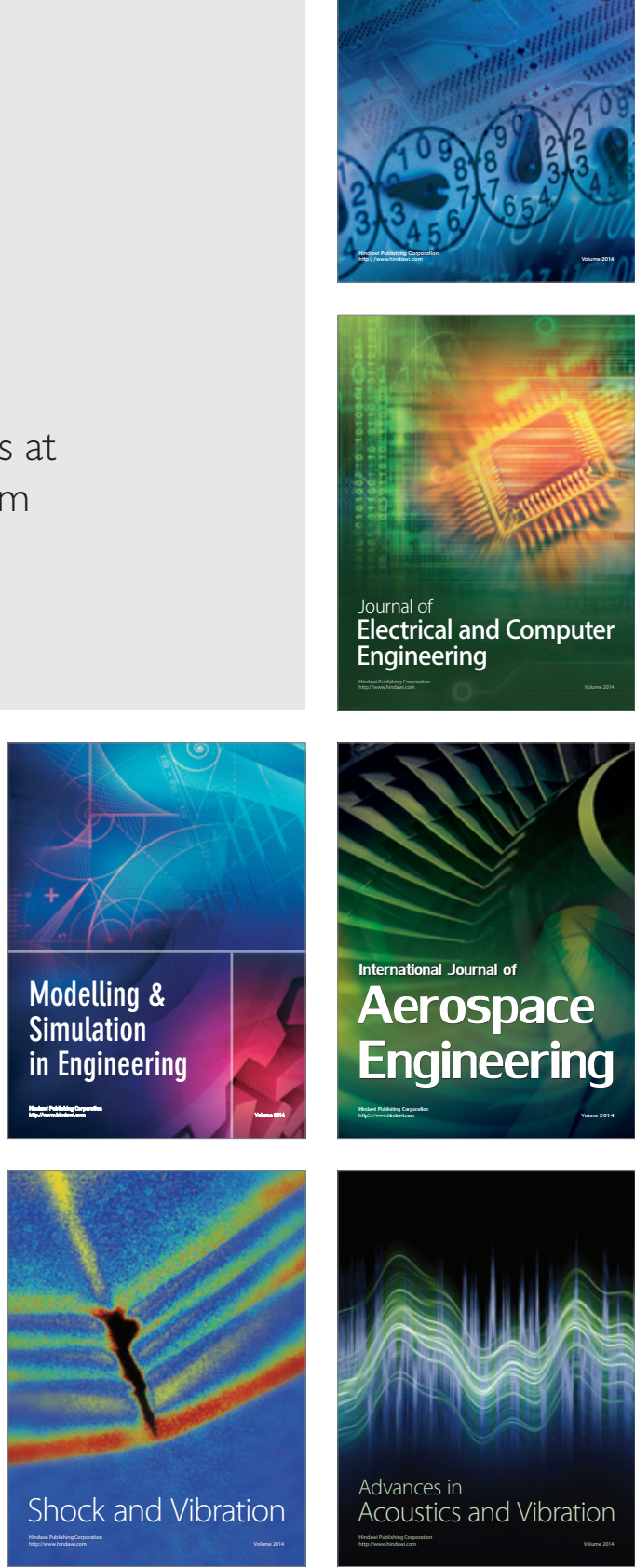